\title{
Identification of excited states in ${ }_{52}^{107} \mathrm{Te}_{55}$
}

W. Zhang $\odot,{ }^{1,{ }^{*}}$ B. Cederwall, ${ }^{1}$ C. Qi,${ }^{1}$ A. Ertoprak,${ }^{1}$ Ö. Aktas, ${ }^{1}$ X. Liu, ${ }^{1}$ K. Andgren,,${ }^{1}$ K. Auranen, ${ }^{2}$ T. Bäck, ${ }^{1}$ L. Barber ${ }^{3}$ G. Beeton, ${ }^{4}$ D. M. Cullen, ${ }^{3}$ I. G. Darby, ${ }^{5}$ M. R. Dimmock, ${ }^{6}$ S. Eeckhaudt, ${ }^{2}$ E. Ganioğlu, ${ }^{7}$ M. Górska ${ }^{8}$ T. Grahn, ${ }^{2}$

P. T. Greenlees, ${ }^{2}$ B. Hadinia, ${ }^{1}$ E. Ideguchi, ${ }^{9}$ A. Illana, ${ }^{2}$ P. M. Jones, ${ }^{2}$ D. T. Joss, ${ }^{6}$ R. Julin, ${ }^{2}$ S. Juutinen, ${ }^{2}$ J. M. Keatings, ${ }^{4}$ A. Khaplanov, ${ }^{1}$ F. Kulali, ${ }^{10}$ M. Leino, ${ }^{2}$ M. Luoma, ${ }^{2}$ B. Lv,${ }^{11}$ B. S. Nara Singh, ${ }^{4}$ L. Nelson, ${ }^{6}$ M. Niikura, ${ }^{12}$ M. Nyman, ${ }^{2}$ J. Ojala, ${ }^{2}$ R. D. Page, ${ }^{6}$ J. Pakarinen, ${ }^{2}$ E. S. Paul,${ }^{6}$ C. Petrache, ${ }^{11}$ M. Petri, ${ }^{13}$ P. Rahkila,${ }^{2}$ P. Ruotsalainen, ${ }^{2}$ M. Sandzelius, ${ }^{2}$ J. Sarén, ${ }^{2}$ C. Scholey, ${ }^{2}$ J. F. Smith, ${ }^{4}$ J. Sorri, ${ }^{2}$ H. Tann, ${ }^{2,6}$ G. Zimba, ${ }^{2}$ J. Uusitalo, ${ }^{2}$ R. Wadsworth, ${ }^{13}$ and R. Wyss ${ }^{1}$

${ }^{1}$ KTH Royal Institute of Technology, 10691 Stockholm, Sweden

${ }^{2}$ Department of Physics, University of Jyväskylä, FIN-40014 Jyväskylä, Finland

${ }^{3}$ Department of Physics and Astronomy, Schuster Building, The University of Manchester, Manchester M13 9PL, United Kingdom

${ }^{4}$ School of Computing Engineering and Physical Sciences, University of the West of Scotland, Paisley PA1 2BE, United Kingdom

${ }^{5}$ SUPA, School of Physics and Astronomy, University of Glasgow, Glasgow G12 8QQ, United Kingdom

${ }^{6}$ Department of Physics, Oliver Lodge Laboratory, University of Liverpool, Liverpool L69 7ZE, United Kingdom

${ }^{7}$ Department of Physics, Istanbul University, 34134 Istanbul, Turkey

${ }^{8}$ GSI Helmholtzzentrum für Schwerionenforschung GmbH, 64291 Darmstadt, Germany

${ }^{9}$ Research Center for Nuclear Physics, Osaka University, JP-567-0047 Osaka, Japan

${ }^{10}$ Nuclear Technology and Radiation Safety Department, Üsküdar University, Istanbul, Turkey

${ }^{11}$ Université Paris-Saclay, CNRS/IN2P3, IJCLab, 91405 Orsay, France

${ }^{12}$ Center for Nuclear Study, University of Tokyo, Wako, Saitama 351-0198, Japan

${ }^{13}$ Department of Physics, University of York, Heslington, York YO10 5DD, United Kingdom

(Received 31 August 2021; accepted 22 November 2021; published 8 December 2021)

\begin{abstract}
Excited states in the extremely neutron-deficient nucleus ${ }^{107} \mathrm{Te}$ have been identified from two separate experiments using the recoil-decay tagging technique. Two connected structures were observed on the basis of $\gamma \gamma$-coincidence relations and tentatively assigned as built on the mixed-parentage $v g_{7 / 2} d_{5 / 2}$ and $v h_{11 / 2}$ intruder configurations. The observed structures were compared with large-scale shell-model calculations and total Routhian surface calculations. Collective behavior was discovered to persist in the $v h_{11 / 2}$ band of ${ }^{107} \mathrm{Te}$ which highlights the shape-polarizing effect of a single valence neutron occupying the $h_{11 / 2}$ intruder orbit as the $N=50$ shell closure is approached.
\end{abstract}

DOI: 10.1103/PhysRevC.104.064305

\section{INTRODUCTION}

The structures of nuclei close to the presumed doubly magic nucleus ${ }^{100} \mathrm{Sn}$ have been the subject of numerous experimental and theoretical studies during the past two decades [1]. The region of nuclei with a few particles outside the ${ }^{100} \mathrm{Sn}$ core, in particular, provides a special "laboratory" for the observation of the competition between single-particle and collective degrees of freedom. In this region, a small change in the number of valence particles can introduce dramatic changes in the nuclear structure.

*Corresponding author: wezh@kth.se

Published by the American Physical Society under the terms of the Creative Commons Attribution 4.0 International license. Further distribution of this work must maintain attribution to the author(s) and the published article's title, journal citation, and DOI. Funded by Bibsam.
The nearly equidistant energy spacings in the ground-state bands of even-mass Te isotopes and the $v h_{11 / 2}$ bands of odd-mass Te isotopes between $N=56$ and $70[2,3]$ indicate that tellurium isotopes may be among the best examples of collective quadrupole vibrations in nuclei. However, the most neutron-deficient tellurium isotope for which excited states have been identified to date, ${ }^{106} \mathrm{Te}$, shows a sudden decrease of energy in the $6^{+} \rightarrow 4^{+}$transition, which is explained to be a senioritylike ground-state band structure [2]. This is quite different from the vibrational-like patterns observed in the low-lying excited states of the heavier tellurium isotopes [4-6]. In the neighboring xenon isotopes [7,8], an enhanced collectivity was seen and interpreted as partially an effect of the enhanced neutron-proton interactions $[9,10]$. The underlying mechanism behind the abrupt change of structure between ${ }^{106} \mathrm{Te}$ [2] and ${ }^{108} \mathrm{Te}$ [5] is not easily explained by theoretical models to date. Experimental information on the structure of ${ }^{107} \mathrm{Te}$ may shed light on this effect and elucidate the mechanisms behind the development of quadrupolecollective structures close to the $N=Z=50$ shell closures. 
In addition to the quadrupole-collective degrees of freedom, octupole correlations have been predicted to maximize in ${ }^{112} \mathrm{Ba}$ and could also play an important role in neighboring $\mathrm{Xe}$ and Te nuclei [11], due to $\Delta l=\Delta j=3$ residual interactions between $1 d_{5 / 2}$ and $0 h_{11 / 2}$ subshells. Experimental evidence for octupole collectivity has been identified from the intermediate-spin states of the xenon isotopes ${ }^{112} \mathrm{Xe}$ [12] and ${ }^{114} \mathrm{Xe}$ [13] and the tellurium isotopes ${ }^{108} \mathrm{Te}[5],{ }^{109} \mathrm{Te}$ [14], and ${ }^{110} \mathrm{Te}$ [15]. Its limit of existence in this region still remains an open question to be explored experimentally. Additional interest in the nucleus ${ }^{107} \mathrm{Te}$ stems from astrophysical considerations since it has been predicted to constitute the endpoint of the rapid-proton-capture process leading to a closed $\mathrm{Sn}-\mathrm{Sb}$ Te cycle $[16,17]$. It is still extremely challenging to directly measure the ${ }^{106} \mathrm{Sb}(p, \gamma)$ reaction rate, and experimental information on the level structure of the ${ }^{107} \mathrm{Te}$ above the proton threshold might contribute to constraining this rate [17].

In this paper, we report a new spectroscopic study of ${ }^{107} \mathrm{Te}$, which significantly extends the limited information on the excited states of ${ }^{107} \mathrm{Te}$ reported previously [18].

\section{EXPERIMENTAL DETAILS}

Excited states in ${ }^{107} \mathrm{Te}$ were populated in two separate experiments performed at the Accelerator Laboratory of the University of Jyväskylä, Finland. Both experiments employed fusion-evaporation reactions and the recoil-decay tagging (RDT) technique $[19,20]$ in order to select events associated with the population of excited states in the rare ${ }^{107} \mathrm{Te}$ products. The first experiment was primarily aimed at identifying excited states of ${ }^{110} \mathrm{Xe}$ [7]. This experiment used a ${ }^{54} \mathrm{Fe}$ beam at $195 \mathrm{MeV}$ to bombard an isotopically enriched $(99.8 \%){ }^{58} \mathrm{Ni}$ target consisting of a $1.0 \mathrm{mg} / \mathrm{cm}^{2}$ self-supporting foil. The average beam intensity was 5 pnA during 5 days of irradiation time. Prompt $\gamma$ rays were detected at the target position by the Jurogam $\gamma$-ray spectrometer consisting of 43 Eurogam Phase I [21] and GASP [22] type Compton-suppressed highpurity germanium detectors. The recoiling fusion evaporation products were separated in-flight from the beam particles by the gas-filled recoil separator RITU $[23,24]$ and implanted into two double-sided silicon strip detectors (DSSDs) of the GREAT spectrometer [25]. Each DSSD has an effective area of $60 \times 40 \mathrm{~mm}^{2}$ and a strip pitch of $1 \mathrm{~mm}$ in both directions, thus yielding 4800 independent pixels in total.

The second experiment was carried out using the inverse reaction ${ }^{54} \mathrm{Fe}\left({ }^{58} \mathrm{Ni}, 2 p 3 n\right){ }^{107} \mathrm{Te}$. The ${ }^{58} \mathrm{Ni}$ ions were accelerated to an energy of $215 \mathrm{MeV}$ and then used to bombard the target foils consisting of $99.9 \%$ isotopically enriched ${ }^{54} \mathrm{Fe}$ with an areal density of $0.77 \mathrm{mg} / \mathrm{cm}^{2}$. The beam intensity varied between 3 and $4 \mathrm{pnA}$ during 12 days of irradiation time. The upgraded Jurogam III $\gamma$-ray spectrometer [26], consisting of 15 Eurogam Phase I-type [21] and 24 Euroball clover [27] escape-suppressed detectors, was used to detect prompt $\gamma$ rays at the target position. The fusion-evaporation products were separated by the new vacuum-mode recoil separator MARA (mass analyzing recoil apparatus) [28,29], which is a complementary device to RITU and can separate the reaction products from the primary beam in symmetric and inverse kinematics more effectively [29]. At the focal plane of

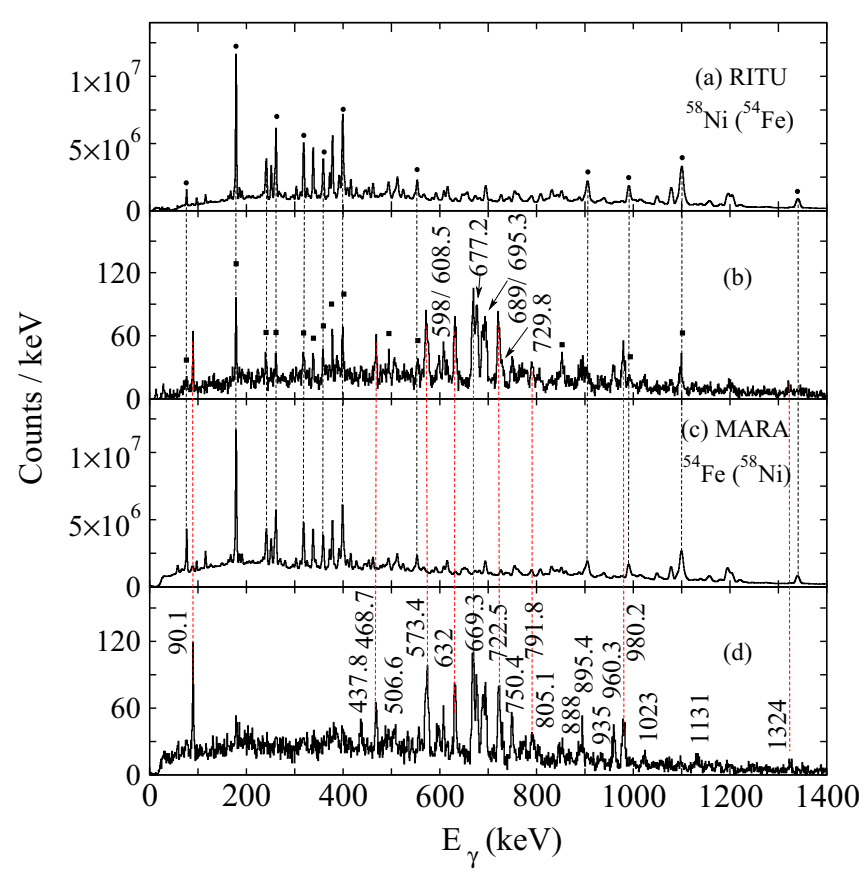

FIG. 1. (a) Recoil-correlated $\gamma$-ray energy spectrum from the RITU experiment, dominated by transitions in the strongest threeproton evaporation reaction channel, ${ }^{109} \mathrm{Sb}$ [35]. (b) As in panel (a), with additional selection on the characteristic $\alpha$-decay energy of ${ }^{107} \mathrm{Te}$. Contamination lines arising from ${ }^{109} \mathrm{Sb}$ are indicated by filled squares. (c) Recoil-correlated $\gamma$-ray energy spectrum from the MARA experiment. (d) RDT $\gamma$-ray spectrum for ${ }^{107} \mathrm{Te}$. The energy calibration of the germanium detectors was performed offline using in-beam $\gamma$ rays of ${ }^{109} \mathrm{Sb}$ for both experiments, indicated by solid circles. The black (and red) dashed lines are drawn to guide the eye and illustrate the energy consistency in the calibration (and $\gamma$ rays for ${ }^{107} \mathrm{Te}$ ) from the two experiments.

MARA, the fusion residues were passed through a multiwire proportional counter and were then implanted into the DSSD of model BB20, which has 72 strips on the $y$ plane and 192 strips on the $x$ plane, each with a width of $0.67 \mathrm{~mm}$ [29].

In both experiments, the signals from all detectors were time-stamped using a 100-MHz clock and recorded independently by the triggerless total data readout acquisition system [30]. The data were sorted online and offline using the GRAIN software package [31]. The $\alpha$ decay of ${ }^{107} \mathrm{Te}$ has been reported with $E_{\alpha}=3.862(10) \mathrm{MeV}$ [32], $b_{\alpha}=70(30) \%$ [33], and $T_{1 / 2}=3.6(2) \mathrm{ms}$ [34], which is short enough to allow clean selections with the highly selective RDT technique $[19,20]$. The search time between a recoil implant and its $\alpha$ decay was limited to $11 \mathrm{~ms}$, and in addition, a gate on the $3.862-\mathrm{MeV} \alpha$ energy was set to select prompt $\gamma$ rays associated with ${ }^{107} \mathrm{Te}$.

\section{RESULTS}

Energy spectra for the prompt $\gamma$ rays recorded at the target position in delayed coincidence with detected recoils in the DSSDs from both experiments are shown in Figs. 1(a) and 1(c), respectively. Figures 1(b) and 1(d) show the RDT $\gamma$ spectra for weakly populated $(\sigma \approx 1 \mu \mathrm{b}){ }^{107} \mathrm{Te}$ from both 


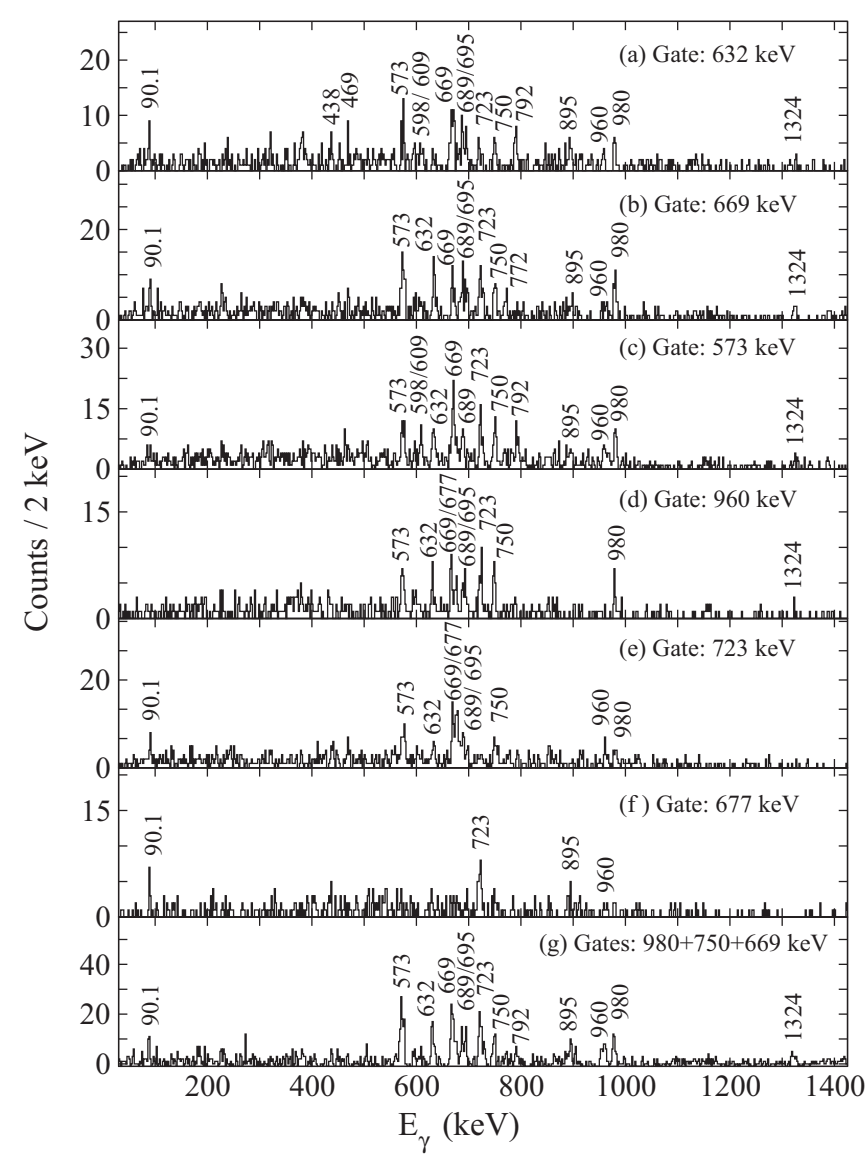

FIG. 2. Coincidence $\gamma$-ray spectra obtained by gating on the (a) 632-keV, (b) 669-keV, (c) 573-keV, (d) $960-\mathrm{keV}$, (e) $723-\mathrm{keV}$, and (f) 677-keV transitions. Panel (g) shows a sum of gates on some transitions in the $\gamma \gamma$ matrix, specifically the $980-, 750-$, or $669-\mathrm{keV}$ transitions.

experiments, illustrating the selective power of the RDT technique. It is worth noting that the contamination from stronger reaction channels has been more efficiently suppressed in Fig. 1(d) from the MARA separator compared with Fig. 1(b) from the RITU separator. However, in the offline analysis for the MARA experiment, in order to maintain statistics, the mass spectrum (mass/charge ratio, $A / q$ ) was not used for the selection and subsequent analysis.

In order to construct a level scheme for ${ }^{107} \mathrm{Te}$, a recoildecay tagged $\gamma \gamma$-coincidence matrix was produced using the combined data from both experiments. Examples of gated coincidence spectra are presented in Fig. 2. Based on coincidence relationships as well as intensity arguments, the deduced level scheme of ${ }^{107} \mathrm{Te}$ is shown in Fig. 3. The tentative spin and parity assignments for the shown levels rely on systematic trends in neighboring odd-mass Te isotopes. The measured energies and relative intensities of the $\gamma$ rays are listed in Table I. Due to the limited statistics and the presence of energy doublets, only about half of the observed $\gamma$ rays listed in Table I could be placed in the level scheme. The low statistics also precluded firm multipolarity assignment by means of angular distribution measurements.

The ground state of ${ }^{107} \mathrm{Te}$ has previously been assigned to have spin-parity $\left(5 / 2^{+}\right)$as being built on a $v d_{5 / 2}$ config-

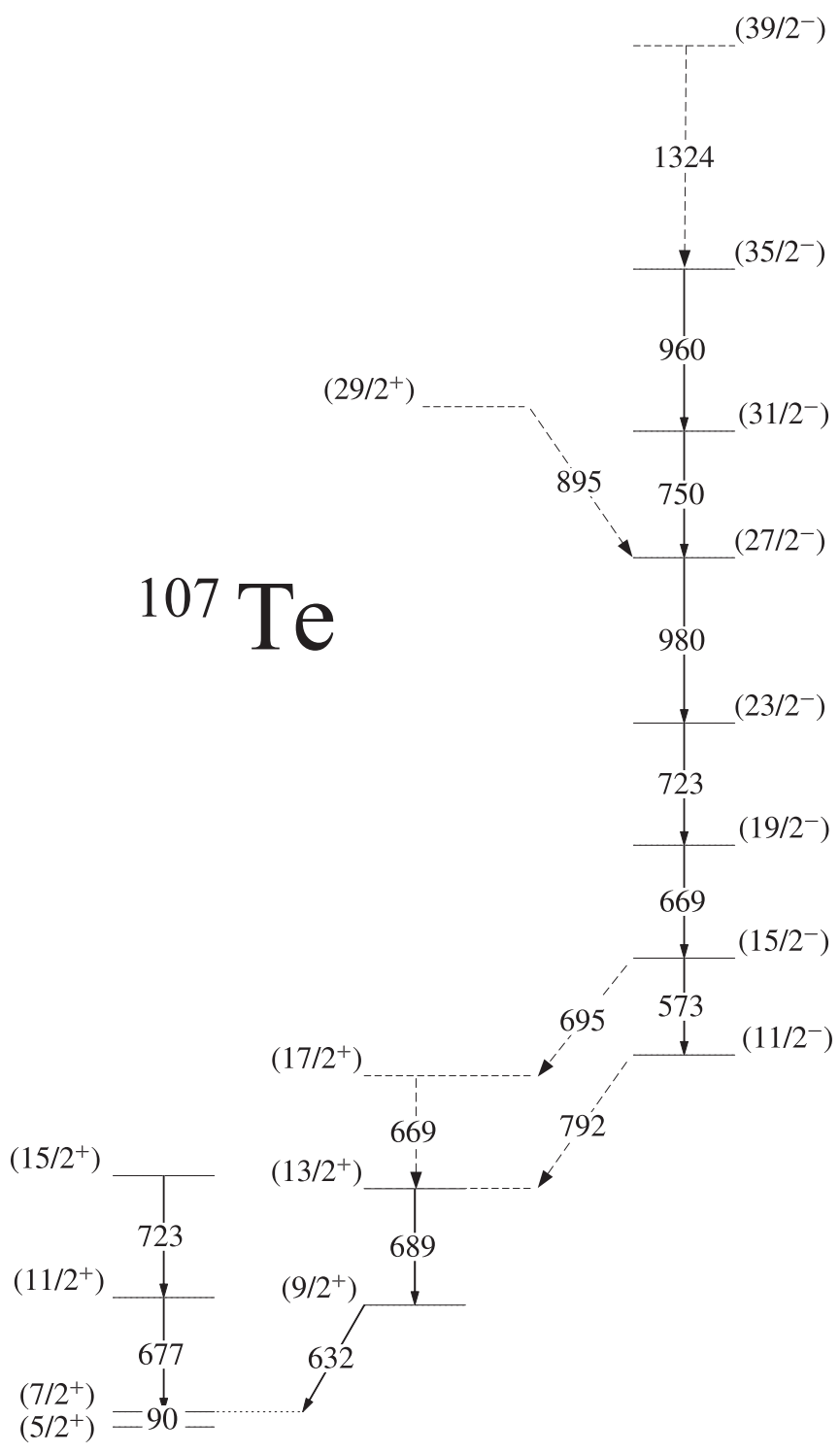

FIG. 3. A tentative level scheme deduced for ${ }^{107} \mathrm{Te}$ from this work. The transition energies are given in $\mathrm{keV}$.

uration, and the $90-\mathrm{keV}$ transition connects the first excited $\left(7 / 2^{+}\right)$state with the ground state [18]. This continues the decreasing trend of the first excited states in the odd-mass tellurium isotopes from $117 \mathrm{keV}$ in ${ }^{111} \mathrm{Te}$ [36] to $98 \mathrm{keV}$ in ${ }^{109} \mathrm{Te}$ [14]. Hadinia et al. [18] also tentatively proposed that the $632-\mathrm{keV}$ transition deexcites a $\left(9 / 2^{+}\right)$state to the first excited $\left(7 / 2^{+}\right)$state and that the $723-\mathrm{keV}$ transition deexcites the same $\left(9 / 2^{+}\right)$state to the ground state. The coincidence relationship between the 632- and 90-keV transitions is confirmed in the present work, as shown in the spectrum in Fig. 2(a). However, the previously suggested placement of the $723-\mathrm{keV}$ transition could not be confirmed. Spectra in Figs. 2(b)-2(f) show the $\gamma$ rays that are in coincidence with the 669-, 573-, 960-, 723-, and 677-keV $\gamma$ rays, respectively. Figure 2(g) shows a coincidence spectrum gated on the 980-, 669-, or 750-keV transitions in the RDT-gated $\gamma \gamma$ matrix. Strong selfcoincident doublets occur for both the 573- and 669-keV $\gamma$ 
TABLE I. Measured energies $\left(E_{\gamma}\right)$ and relative intensities $\left(I_{\text {rel }}\right.$, without consideration of internal conversion) for $\gamma$-ray transitions in ${ }^{107} \mathrm{Te}$ observed in this work. The initial and final spins and parities $\left(J_{i}^{\pi_{i}}\right.$ and $\left.J_{f}^{\pi_{f}}\right)$, where given in the third column, are tentatively assigned from systematics.

\begin{tabular}{|c|c|c|}
\hline$E_{\gamma}(\mathrm{keV})$ & $I_{\text {rel }}$ & $J_{i}^{\pi} \rightarrow J_{f}^{\pi}$ \\
\hline $90.1(1)^{\mathrm{a}}$ & $100(8)$ & $\left(7 / 2^{+}\right) \rightarrow\left(5 / 2^{+}\right)$ \\
\hline $437.8(8)$ & $17(5)$ & \\
\hline $468.7(4)^{*}$ & $34(5)$ & \\
\hline $506.6(8)$ & $16(6)$ & \\
\hline $573.4(3)^{\mathrm{a}, *}$ & $119(8)$ & $\left(15 / 2^{-}\right) \rightarrow\left(11 / 2^{-}\right)$ \\
\hline $598.0(7)$ & $24(5)$ & \\
\hline $608.5(4)$ & $31(5)$ & \\
\hline $632.0(3)^{\mathrm{a}}$ & $76(6)$ & $\left(9 / 2^{+}\right) \rightarrow\left(7 / 2^{+}\right)$ \\
\hline $669.3(3)^{\mathrm{a}, *}$ & $124(8)$ & $\begin{array}{l}\left(19 / 2^{-}\right) \rightarrow\left(15 / 2^{-}\right) \\
\left(17 / 2^{+}\right) \rightarrow\left(13 / 2^{+}\right)\end{array}$ \\
\hline $677.2(3)^{\mathrm{a}}$ & $96(8)$ & $\left(11 / 2^{+}\right) \rightarrow\left(7 / 2^{+}\right)$ \\
\hline $689.0(3)^{\mathrm{a}}$ & $72(7)$ & $\left(13 / 2^{+}\right) \rightarrow\left(9 / 2^{+}\right)$ \\
\hline $695.3(4)^{\mathrm{a}, *}$ & $80(8)$ & $\left(15 / 2^{-}\right) \rightarrow\left(17 / 2^{+}\right)$ \\
\hline $722.5(5)^{\mathrm{a}, *}$ & $98(3)$ & $\begin{array}{l}\left(23 / 2^{-}\right) \rightarrow\left(19 / 2^{-}\right) \\
\left(15 / 2^{+}\right) \rightarrow\left(11 / 2^{+}\right)\end{array}$ \\
\hline $729.8(8)$ & $24(5)$ & \\
\hline $750.4(6)$ & $47(6)$ & $\left(31 / 2^{-}\right) \rightarrow\left(27 / 2^{-}\right)$ \\
\hline $772(2)$ & $15(10)$ & \\
\hline $778(2)$ & $18(11)$ & \\
\hline $791.8(5)$ & $37(5)$ & $\left(11 / 2^{-}\right) \rightarrow\left(13 / 2^{+}\right)$ \\
\hline $805.1(9)$ & $16(5)$ & \\
\hline $888(1)$ & $19(5)$ & \\
\hline $895.4(7)$ & $32(6)$ & $\left(29 / 2^{+}\right) \rightarrow\left(27 / 2^{-}\right)$ \\
\hline $935(1)$ & $11(4)$ & \\
\hline $960.3(4)$ & $45(5)$ & $\left(35 / 2^{-}\right) \rightarrow\left(31 / 2^{-}\right)$ \\
\hline $980.2(4)$ & $74(6)$ & $\left(27 / 2^{-}\right) \rightarrow\left(23 / 2^{-}\right)$ \\
\hline 1023.1(9) & $16(4)$ & \\
\hline 1131(1) & $17(5)$ & \\
\hline $1324(1)$ & $17(4)$ & $\left(39 / 2^{-}\right) \rightarrow\left(35 / 2^{-}\right)$ \\
\hline
\end{tabular}

a Transitions also observed in Ref. [18].

*The stars denote doublet transitions.

rays, which cannot be resolved experimentally. With reference to the coincidence relationships shown in Fig. 2, a set of mutually coincident transitions with energies 632, 689, 573, $669,723,980,750$, and $960 \mathrm{keV}$ is observed. Based on the fact that the $689-\mathrm{keV} \gamma$-ray transition is strongly correlated with the $632-\mathrm{keV}$ transition but relatively more weakly correlated with the 573- and 960- keV transitions, the 689-keV line has been assigned as depopulating the $\left(13 / 2^{+}\right)$state into the $\left(9 / 2^{+}\right)$state.

In the nearby heavier odd- $A$ Te isotopes, the level schemes are dominated by a yrast sequence which is built on the $v h_{11 / 2}$ single-particle configuration. The systematic behavior in the yrast sequences shown in Fig. 4 agrees with the placement of the $573-\mathrm{keV}$ transition as depopulating the $\left(15 / 2^{-}\right)$state to the $\left(11 / 2^{-}\right)$state and the $669-\mathrm{keV}$ transition deexciting the $\left(19 / 2^{-}\right)$state to the $\left(15 / 2^{-}\right)$state. The ordering of the other four transitions built on the $v h_{11 / 2}$ yrast band in Fig. 3 is based on their relative $\gamma$-ray intensities and is less certain due to the presence of doublet transitions. Two other $\gamma$ rays, i.e., the 895and 1324-keV transitions shown in Fig. 2(g), were found to be

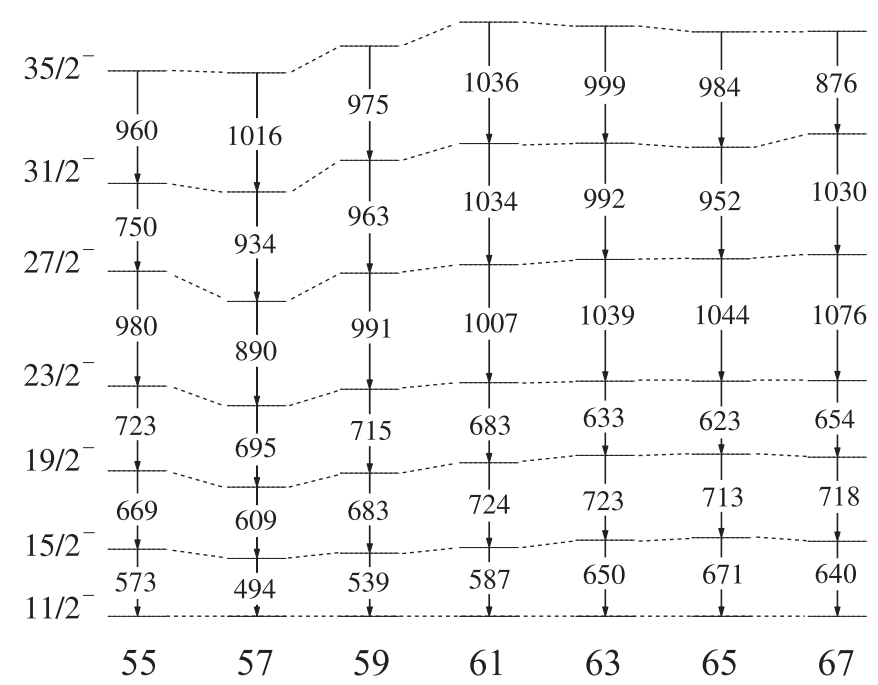

FIG. 4. Excitation energies of states in the yrast $v h_{11 / 2}$ bands in the odd- $A$ tellurium isotopes, relative to the respective $11 / 2^{-}$bandheads. The data for heavier Te isotopes are taken from Refs. [3,14,36] and the data for ${ }^{107} \mathrm{Te}$ are derived from the present work. The dashed lines are drawn to show the energy evolution as the number of neutrons changes.

weakly coincident with the lower-spin members of the $h_{11 / 2}$ band. Their placements are tentatively assigned and indicated by the dashed lines in Fig. 3. As the neutron number decreases towards the $N=50$ shell gap, the $v h_{11 / 2}$ state increases to a higher energy relative to the ground state, from an excitation energy of $280 \mathrm{keV}$ in ${ }^{115} \mathrm{Te}$ [37] to $839 \mathrm{keV}$ in ${ }^{111} \mathrm{Te}$ [36] and then to $1089 \mathrm{keV}$ in ${ }^{109} \mathrm{Te}$ [14]. In ${ }^{109,111} \mathrm{Te}$, this $v h_{11 / 2}$ state decays through the $9 / 2^{+}$and $7 / 2^{+}$states to the $5 / 2^{+}$ground state. In particular, in the case of ${ }^{109} \mathrm{Te}$, several decay paths have been observed [14]. Such decay paths in ${ }^{107} \mathrm{Te}$ could not be firmly determined in the present work. Linking transitions between the negative-parity structure and the low-lying states are tentatively assigned and indicated by the dashed lines in Fig. 3.

The 677-keV transition has a relatively high intensity but is only observed in strong coincidence with the 723 - and $90-\mathrm{keV}$ transitions, as shown in Fig. 2(f). This 723-keV transition is not the same transition as that one in the $v h_{11 / 2}$ yrast band, indicating that the $723-\mathrm{keV}$ transition is also a doublet. This could be confirmed from the spectrum in Fig. 2(d) where the $960-\mathrm{keV}$ transition is in coincidence with the $723-\mathrm{keV}$ transition much more strongly than with the $677-\mathrm{keV}$ transition. Considering the systematic trends of the positive-parity states in ${ }^{109,111} \mathrm{Te}[14,36]$, the 677 - and $723-\mathrm{keV}$ transitions are tentatively assigned to a positive-parity structure in ${ }^{107} \mathrm{Te}$.

\section{DISCUSSION}

The systematics of the yrast $v h_{11 / 2}$ bands of the neutrondeficient odd- $A$ tellurium isotopes with $N<68$ are shown in Fig. 4, in which the excitation energies of states are given relative to the respective $11 / 2^{-}$bandheads. It can be seen that the excitation energies of states within the $v h_{11 / 2}$ bands vary smoothly as a function of the neutron number $N$. As the 


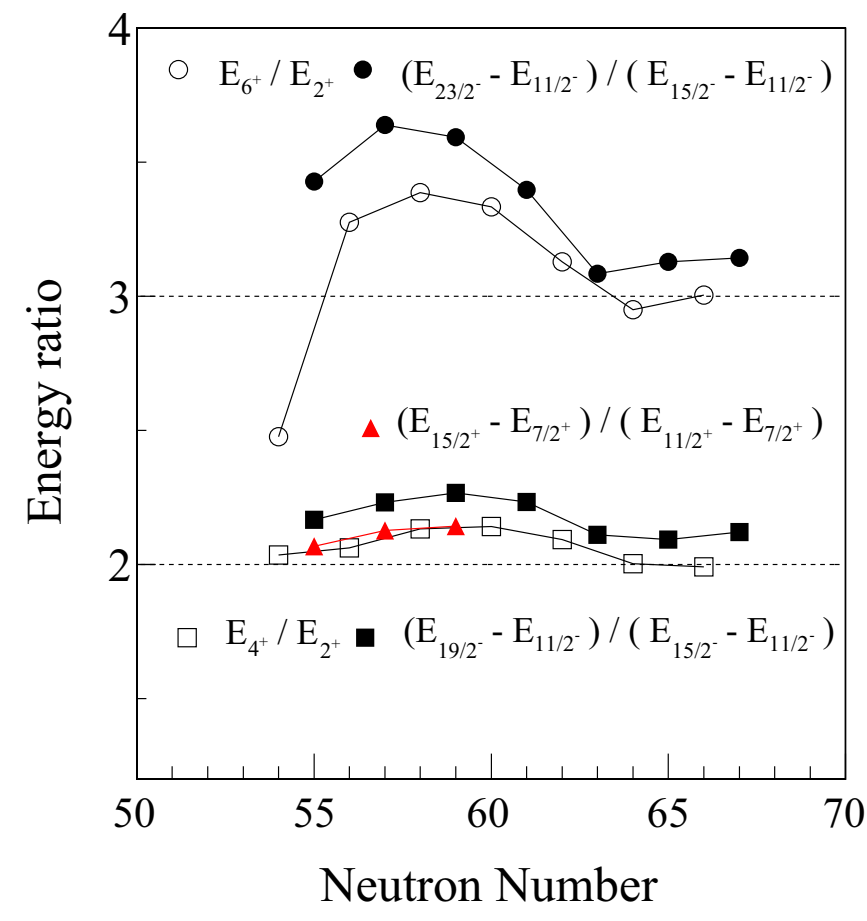

FIG. 5. Energy ratios plotted versus $N$ for even- and odd- $A{ }_{52} \mathrm{Te}$ isotopes in the mass region $A \leqslant 119$. The data are taken from the present work and Refs. [2,3,5,14,15,18,36].

neutron number is reduced from around the midshell at $N=$ 65 , the energy of the $15 / 2^{-} \rightarrow 11 / 2^{-}$transition decreases until $N=57\left({ }^{109} \mathrm{Te}\right)$, where it reaches a minimum, and then its expected increasing trend is eventually restored at ${ }^{107} \mathrm{Te}$. This tendency follows the behavior analogous to that in the more extensively studied even- $A$ tellurium isotopes [2], of which the increasing trend of the $E\left(2^{+}\right)$energy is restored at ${ }^{106} \mathrm{Te}$. To elucidate the structural evolution in the tellurium isotopic chain, excitation energy ratios, $E_{4_{1}^{+}} / E_{2_{1}^{+}}$and $E_{6_{1}^{+}} / E_{2_{1}^{+}}$for even$A$ Te isotopes, and $\left(E_{19 / 2^{-}}-E_{11 / 2^{-}}\right) /\left(E_{15 / 2^{-}}-E_{11 / 2^{-}}\right)$and $\left(E_{23 / 2^{-}}-E_{11 / 2^{-}}\right) /\left(E_{15 / 2^{-}}-E_{11 / 2^{-}}\right)$energy difference ratios for odd- $A$ Te isotopes with $N<68$, are shown in Fig. 5. These energy ratios are valuable indicators of nuclear deformation and collectivity, and compared with the predictions for a harmonic vibrator, $E_{4_{1}^{+}} / E_{2_{1}^{+}}=2$ and $E_{6_{1}^{+}} / E_{2_{1}^{+}}=3$ as indicated by dashed lines.

Even- $A$ Te isotopes are usually considered to be vibrational at low spin inferred from the energy ratios $E_{4_{1}^{+}} / E_{2_{1}^{+}} \approx 2$. The energy ratios of odd-mass Te isotopes appear to follow the same trends as the even-mass Te isotopes. As the neutron number decreases from the midshell $(N=66)$ towards the $N=50$ shell closure, these ratios for both even- $A$ and odd $-A$ Te isotopes show a noticeable deviation from the harmonic limits, in contrast to the smoothly decreasing behavior of even-mass tellurium isotopes when approaching $N=82$ [2]. This distinctive deviation is firstly reminiscent of enhanced collectivity. However, from lifetime measurements of the first $2^{+}$excited states in the neighboring even- $A$ Te isotopes $\left({ }^{108} \mathrm{Te}\right.$ [38], ${ }^{110} \mathrm{Te}$ [39], ${ }^{112} \mathrm{Te}$ [40]), it is found that the measured $B\left(E 2 ; 2_{\mathrm{gs}}^{+} \rightarrow 0^{+}\right)$values show an apparent decrease as $N$ decreases, indicating that these isotopes do not show any enhanced transition probabilities. In the most neutron-deficient tellurium isotopes, neutrons and protons are predicted to occupy the same sets of orbitals, i.e., the near-degenerate $1 d_{5 / 2}$ and $0 g_{7 / 2}$ subshells, and the $0 h_{11 / 2}$ intruder state at relatively higher excitation energy, and thus enhanced neutron-proton correlations are expected to come into play when approaching the $N=Z$ line. This uncoordinated relationship between excitation energies and $B(E 2)$ values can be associated with the effect of enhanced neutron-proton pairing. This intriguing phenomenon has been investigated in several theoretical calculations using the quasiparticle random-phase approximation model [10] and the shell model [41,42], revealing that excitation energies are more sensitive to pairing than the $B(E 2)$ values [41].

Figure 5 also shows the energy ratios $\left(E_{15 / 2^{+}}-\right.$ $\left.E_{7 / 2^{+}}\right) /\left(E_{11 / 2^{+}}-E_{7 / 2^{+}}\right)$in the positive-parity bands of ${ }^{107} \mathrm{Te}$, ${ }^{109} \mathrm{Te}$, and ${ }^{111} \mathrm{Te}$. These ratios are well interpolated into the ratio curve of even- $A$ Te isotopes. However, the energy ratios of the $v h_{11 / 2}$ bands in odd $A$ Te isotopes are generally higher than those of the ground-state bands in neighboring even- $A$ Te isotopes, implying that the negative-parity $v h_{11 / 2}$ bands have an appreciably stronger collectivity and larger deformations. In addition, the measured $B\left(E 2 ; 15 / 2^{-} \rightarrow 11 / 2^{-}\right)$value in the $v h_{11 / 2}$ band of ${ }^{109} \mathrm{Te}$ is larger than the $B\left(E 2 ; 0_{\mathrm{gs}}^{+} \rightarrow 2^{+}\right)$ value in the ground band of ${ }^{108}$ Te by almost a factor of 2.5 [43], and the measured $B(E 2)$ value in the positive-parity band of ${ }^{109} \mathrm{Te}$ is approximately equal to the $B\left(E 2 ; 0_{\mathrm{gs}}^{+} \rightarrow 2^{+}\right)$value of ${ }^{108} \mathrm{Te}$ [43]. Such a significant enhancement of collectivity in the negative-parity bands was attributed to a deformation driving force by the polarization of the valence $h_{11 / 2}$ neutron $[36,43,44]$. This polarization seems to persist in the $v h_{11 / 2}$ band of ${ }^{107} \mathrm{Te}$, but the presence of such a relatively strong collectivity is unexpected as the $N=50$ shell closure is approached. This unusual effect has been previously suggested to arise from increased octupole correlations [12]. However, it is excluded as a possible cause here since the onset of octupole correlations were observed at the intermediate and high spins of $7^{-}$of ${ }^{108} \mathrm{Te}$ [5] and $33 / 2^{+}$of ${ }^{109} \mathrm{Te}$ [14], respectively. An alternative explanation may be related to the enhanced neutron-proton correlations taking place in the presence of neutrons and protons occupying near-identical orbits. This might suggest that in ${ }^{107} \mathrm{Te}$ the polarization of the odd $h_{11 / 2}$ neutron will enlarge the overlap of the neutron-proton wave functions, leading to an increase in the neutron-proton quadrupole-quadrupole interaction strength. One of the consequences is that a single-particle effect like the compression of the $6^{+} \rightarrow 4^{+}$transition energy [2] in ${ }^{106} \mathrm{Te}$ does not appear to be present in the $v h_{11 / 2}$ band of ${ }^{107} \mathrm{Te}$ (see the most striking discrepancy of energy ratios between odd- and even- $A$ Te isotopes in Fig. 5).

To gain further insight into the structures of ${ }^{107} \mathrm{Te}$, largescale shell-model (LSSM) calculations have been performed using a realistic CD-Bonn potential [45], renormalized using the perturbative $G$-matrix approach [46]. The model space included single-particle orbitals $0 g_{7 / 2}, 1 d_{5 / 2}, 1 d_{3 / 2}, 2 s_{1 / 2}$, and $0 h_{11 / 2}$ between the $N, Z=50$ to 82 shell closures (see details in Refs. $[42,47])$. Calculated positive-parity levels are shown 


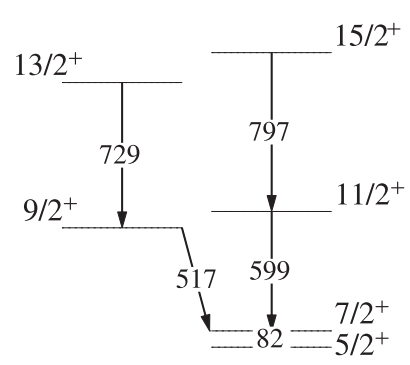

Cal.

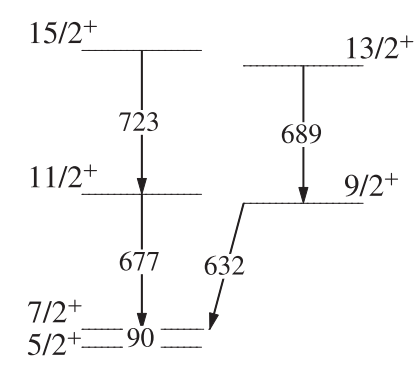

Exp.
FIG. 6. Experimental positive-parity levels in ${ }^{107}$ Te derived from this work in comparison with LSSM calculations. See text for details.

in Fig. 6 in comparison with the experimental results. The calculations are in good agreement with the observed excitation energies. In Fig. 7, the experimental energy levels of the yrast $v h_{11 / 2}$ band of ${ }^{107} \mathrm{Te}$ deduced from the present work are shown in comparison with the ground-state band level energies for the neighboring even-even ${ }^{106} \mathrm{Te}[2]$ and ${ }^{108} \mathrm{Te}$ [5] isotopes and the results from the LSSM calculations. The $v h_{11 / 2}$ band of ${ }^{107} \mathrm{Te}$ reflects a structure more similar to that of the ground-state band of ${ }^{108} \mathrm{Te}$ rather than that of ${ }^{106} \mathrm{Te}$ for which the compression of the $6^{+} \rightarrow 4^{+}$transition energy signals an emerging dominance of single-particle structure. Incidentally, this transition to a less collective ground-state band in ${ }^{106} \mathrm{Te}$ is not well reproduced by the shell-model calculation. In the $v h_{11 / 2}$ band of ${ }^{107} \mathrm{Te}$, it appears that enhanced neutron-proton correlations might suppress the emergence of seniority coupling effectively. Lifetime measurements in the $v h_{11 / 2}$ band of ${ }^{107} \mathrm{Te}$ would be of benefit to examine this effect more conclusively.

To investigate theoretical predictions for the shape-driving effects of the odd $h_{11 / 2}$ neutron, total Routhian surface (TRS) calculations $[48,49]$ were carried out for ${ }^{106,107,108,109} \mathrm{Te}$ in this
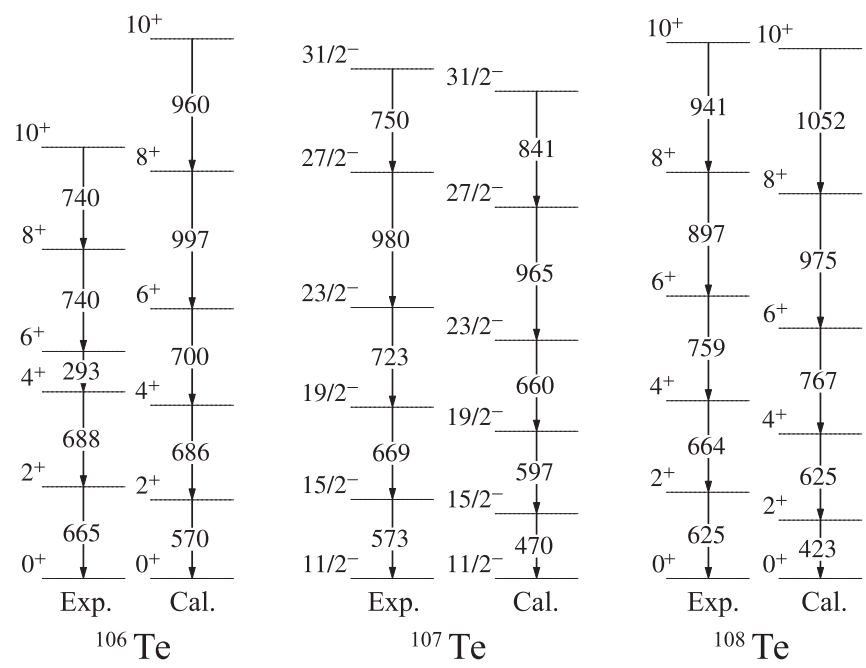

FIG. 7. Excitation energies of states in the yrast $v h_{11 / 2}$ band of ${ }^{107} \mathrm{Te}$ and the ground-state bands of the neighboring even-even ${ }^{106} \mathrm{Te}$ [2] and ${ }^{108} \mathrm{Te}$ [5] isotopes in comparison with the shell-model calculations. See text for details.

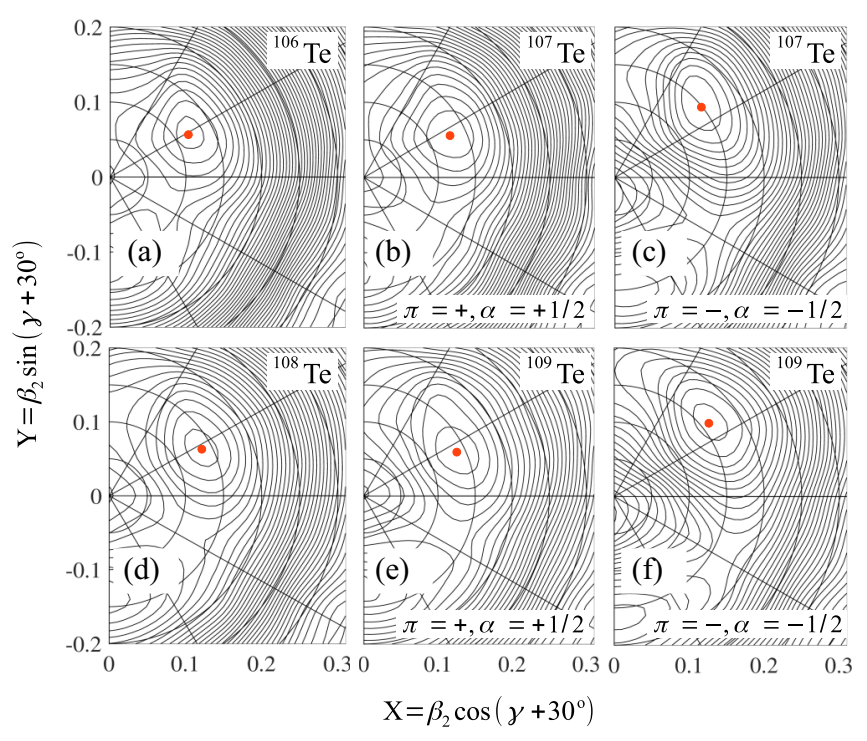

FIG. 8. Calculated TRSs for ground-state bands of ${ }^{106,108} \mathrm{Te}$ and for positive-parity and negative-parity bands of ${ }^{107,109} \mathrm{Te}$ at a rotational frequency of $\hbar \omega=0.2 \mathrm{MeV}$. The energy difference between contour lines is $200 \mathrm{keV}$. The minimum points are indicated by the red dots. Calculated deformation parameters $\left(\beta_{2}, \gamma\right)$ are (a) $(0.12$, $\left.-1^{\circ}\right)$ for ${ }^{106} \mathrm{Te}$; (b) $\left(0.13,-5^{\circ}\right)$ for $(+,+1 / 2)$ and $(\mathrm{c})\left(0.15,10^{\circ}\right)$ for $(-,-1 / 2)$ of ${ }^{107} \mathrm{Te}$; (d) $\left(0.14,-2^{\circ}\right)$ for ${ }^{108} \mathrm{Te}$; and (e) $\left(0.14,-5^{\circ}\right)$ for $(+,+1 / 2)$ and (f) $\left(0.16,9^{\circ}\right)$ for $(-,-1 / 2)$ of ${ }^{109} \mathrm{Te}$.

work. As shown in Fig. 8, TRS calculations demonstrate that a neutron occupying the negative-parity $h_{11 / 2}$ orbital can indeed polarize the quadrupole deformation to a more pronounced value than occupying the positive-parity orbital for both ${ }^{107} \mathrm{Te}$ and ${ }^{109} \mathrm{Te}$ (from $\beta_{2}=0.14$ to $\beta_{2}=0.16$ for ${ }^{109} \mathrm{Te}$ and from $\beta_{2}=0.13$ to $\beta_{2}=0.15$ for ${ }^{107} \mathrm{Te}$ ). There is also a visible effect of the triaxial-shape driving force of the $h_{11 / 2}$ valence neutron in the potential energy surfaces for negative parity, negative signature in Fig. 8. Moreover, the difference in the $\beta_{2}$ value between the positive-parity configuration of ${ }^{107} \mathrm{Te}$ and the ground state of ${ }^{106} \mathrm{Te}$ is very small, and the same is also true for ${ }^{109} \mathrm{Te}$ and ${ }^{108} \mathrm{Te}$. It is therefore concluded that the odd neutron occupying the positive-parity orbital has little effect on either the vibrational collectivity or the nuclear deformation. In addition, it would be of considerable interest to extend the positive-parity bands in ${ }^{107} \mathrm{Te}$ to identify whether the expected seniority coupling will come into play at a higher spin and to build the connections between the negative-parity and positive-parity bands.

\section{CONCLUSIONS}

In summary, excited states in the very neutron-deficient nuclide ${ }^{107} \mathrm{Te}$ were observed for the first time in the present work. Two distinct structures tentatively assigned to the $v h_{11 / 2}$ and $\nu g_{7 / 2}$ quasiparticle states were identified. The structures have been discussed within the context of LSSM and total Routhian surface calculations. It is suggested that a neutron occupying the $h_{11 / 2}$ orbit has a more significant effect on the collectivity than in the positive-parity $d_{5 / 2}$ and $g_{7 / 2}$ orbitals. It is concluded that enhanced neutron-proton correlations might 
play a defining role in the balance between single-particle and collective structures in the Te isotopes when approaching $N=50$.

\section{ACKNOWLEDGMENTS}

This work was supported by the Swedish Research Council under Grant No. 2019-04880; the United Kingdom Science and Technology Facilities Council (STFC) under Grants
No. ST/L005727/1 and No. ST/P003885/1; the EU 7th Framework Programme, Integrating Activities Transnational Access, Project No. 262010 ENSAR; and the Academy of Finland under the Finnish Centre of Excellence Programme (Nuclear and Accelerator Based Physics Programme at JYFL). W.Z. acknowledges support from the China Scholarship Council under Grant No. 201700260239. The authors acknowledge the support of the GAMMAPOOL for the JUROGAM detectors.
[1] T. Faestermann, M. Górska, and H. Grawe, Prog. Part. Nucl. Phys. 69, 85 (2013).

[2] B. Hadinia, B. Cederwall, J. Blomqvist, E. Ganioglu, P. T. Greenlees, K. Andgren, I. G. Darby, S. Eeckhaudt, E. Ideguchi, P. M. Jones, D. T. Joss, R. Julin, S. Juutinen, S. Ketelhut, K. Lagergren, A. P. Leppanen, M. Leino, M. Nyman, J. Pakarinen, E. S. Paul et al., Phys. Rev. C 72, 041303(R) (2005).

[3] https://www.nndc.bnl.gov/.

[4] D. Sohler et al., Eur. Phys. J. A 3, 209 (1998).

[5] G. J. Lane, D. B. Fossan, J. M. Sears, J. F. Smith, J. A. Cameron, R. M. Clark, I. M. Hibbert, V. P. Janzen, R. Krücken, I.-Y. Lee, A. O. Macchiavelli, C. M. Parry, and R. Wadsworth, Phys. Rev. C 57, R1022(R) (1998).

[6] E. S. Paul, A. J. Boston, C. J. Chiara, M. Devlin, D. B. Fossan, S. J. Freeman, D. R. LaFosse, G. J. Lane, M. J. Leddy, I. Y. Lee, A. O. Macchiavelli, P. J. Nolan, D. G. Sarantites, J. M. Sears, A. T. Semple, J. F. Smith, and K. Starosta, Phys. Rev. C 76, 034322 (2007).

[7] M. Sandzelius, B. Hadinia, B. Cederwall, K. Andgren, E. Ganiouglu, I. G. Darby, M. R. Dimmock, S. Eeckhaudt, T. Grahn, P. T. Greenlees, E. Ideguchi, P. M. Jones, D. T. Joss, R. Julin, S. Juutinen, A. Khaplanov, M. Leino, L. Nelson, M. Niikura, M. Nyman et al., Phys. Rev. Lett. 99, 022501 (2007).

[8] L. Capponi, J. F. Smith, P. Ruotsalainen, C. Scholey, P. Rahkila, L. Bianco, A. J. Boston, H. C. Boston, D. M. Cullen, X. Derkx, M. C. Drummond, T. Grahn, P. T. Greenlees, L. Grocutt, B. Hadinia, U. Jakobsson, D. T. Joss, R. Julin, S. Juutinen, M. Labiche et al., Phys. Rev. C 101, 014313 (2020).

[9] E. Caurier, F. Nowacki, A. Poves, and K. Sieja, Phys. Rev. C 82, 064304 (2010).

[10] D. S. Delion, R. Wyss, R. J. Liotta, B. Cederwall, A. Johnson, and M. Sandzelius, Phys. Rev. C 82, 024307 (2010).

[11] J. Skalski, Phys. Lett. B 238, 6 (1990).

[12] J. F. Smith et al., Phys. Lett. B 523, 13 (2001).

[13] G. de Angelis et al., Phys. Lett. B 535, 93 (2002).

[14] A. J. Boston, E. S. Paul, C. J. Chiara, M. Devlin, D. B. Fossan, S. J. Freeman, D. R. LaFosse, G. J. Lane, M. Leddy, I. Y. Lee, A. O. Macchiavelli, P. J. Nolan, D. G. Sarantites, J. M. Sears, A. T. Semple, J. F. Smith, and K. Starosta, Phys. Rev. C 61, 064305 (2000).

[15] E. S. Paul, H. R. Andrews, T. E. Drake, J. DeGraaf, V. P. Janzen, S. Pilotte, D. C. Radford, and D. Ward, Phys. Rev. C 50, R534(R) (1994).

[16] H. Schatz, A. Aprahamian, V. Barnard, L. Bildsten, A. Cumming, M. Ouellette, T. Rauscher, F.-K. Thielemann, and M. Wiescher, Phys. Rev. Lett. 86, 3471 (2001).

[17] V. V. Elomaa, G. K. Vorobjev, A. Kankainen, L. Batist, S. Eliseev, T. Eronen, J. Hakala, A. Jokinen, I. D. Moore, Y. N.
Novikov, H. Penttila, A. Popov, S. Rahaman, J. Rissanen, A. Saastamoinen, H. Schatz, D. M. Seliverstov, C. Weber, and J. Aysto, Phys. Rev. Lett. 102, 252501 (2009).

[18] B. Hadinia, B. Cederwall, K. Lagergren, J. Blomqvist, T. Back, S. Eeckhaudt, T. Grahn, P. Greenlees, A. Johnson, D. T. Joss, R. Julin, S. Juutinen, H. Kettunen, M. Leino, A. P. Leppanen, R. J. Liotta, P. Nieminen, M. Nyman, J. Pakarinen, E. S. Paul et al., Phys. Rev. C 70, 064314 (2004).

[19] E. S. Paul, P. J. Woods, T. Davinson, R. D. Page, P. J. Sellin, C. W. Beausang, R. M. Clark, R. A. Cunningham, S. A. Forbes, D. B. Fossan, A. Gizon, J. Gizon, K. Hauschild, I. M. Hibbert, A. N. James, D. R. LaFosse, I. Lazarus, H. Schnare, J. Simpson, R. Wadsworth et al., Phys. Rev. C 51, 78 (1995).

[20] R. S. Simon et al., Z. Phys. A 325, 197 (1986).

[21] C. W. Beausang et al., Nucl. Instrum. Methods Phys. Res., Sect. A 313, 37 (1992).

[22] C. R. Alvarez et al., Nucl. Phys. News 3, 10 (1993).

[23] M. Leino et al., Nucl. Instrum. Methods Phys. Res., Sect. B 99, 653 (1995).

[24] J. Sarén, J. Uusitalo, M. Leino, and J. Sorri, Nucl. Instrum. Methods Phys. Res., Sect. A 654, 508 (2011).

[25] R. D. Page et al., Nucl. Instrum. Methods Phys. Res., Sect. B 204, 634 (2003).

[26] J. Pakarinen et al., Eur. Phys. J. A 56, 149 (2020).

[27] G. Duchêne et al., Nucl. Instrum. Methods Phys. Res., Sect. A 432, 90 (1999).

[28] J. Sarén et al., Nucl. Instrum. Methods Phys. Res., Sect. A 266, 4196 (2008).

[29] J. Uusitalo, J. Saren, J. Partanen, and J. Hilton, Acta Phys. Pol., B 50, 319 (2019).

[30] I. H. Lazarus et al., IEEE Trans. Nucl. Sci. 48, 567 (2001).

[31] P. Rahkila, Nucl. Instrum. Methods Phys. Res., Sect. A 595, 637 (2008).

[32] F. Heine et al., Z. Phys. A 340, 225 (1991).

[33] D. Schardt et al., Nucl. Phys. A 326, 65 (1979).

[34] K. Auranen et al., Phys. Lett. B 792, 187 (2019).

[35] V. P. Janzen, D. R. LaFosse, H. Schnare, D. B. Fossan, A. Galindo-Uribarri, J. R. Hughes, S. M. Mullins, E. S. Paul, L. Persson, S. Pilotte, D. C. Radford, I. Ragnarsson, P. Vaska, J. C. Waddington, R. Wadsworth, D. Ward, J. Wilson, and R. Wyss, Phys. Rev. Lett. 72, 1160 (1994).

[36] K. Starosta, C. J. Chiara, D. B. Fossan, T. Koike, D. R. LaFosse, G. J. Lane, J. M. Sears, J. F. Smith, A. J. Boston, P. J. Nolan, E. S. Paul, A. T. Semple, M. Devlin, D. G. Sarantites, I. Y. Lee, and A. O. Macchiavelli, Phys. Rev. C 61, 034308 (2000).

[37] T. Lönnroth, A. Virtanen, and J. Hattula, Phys. Scr. 34, 682 (1986). 
[38] T. Bäck, C. Qi, F. Ghazi Moradi, B. Cederwall, A. Johnson, R. Liotta, R. Wyss, H. Al-Azri, D. Bloor, T. Brock, R. Wadsworth, T. Grahn, P. T. Greenlees, K. Hauschild, A. Herzan, U. Jacobsson, P. M. Jones, R. Julin, S. Juutinen, S. Ketelhut et al., Phys. Rev. C 84, 041306(R) (2011).

[39] D. A. Testov et al., Phys. Rev. C 103, 044321 (2021).

[40] M. Doncel, T. Back, D. M. Cullen, D. Hodge, C. Qi, B. Cederwall, M. J. Taylor, M. Procter, K. Auranen, T. Grahn, P. T. Greenlees, U. Jakobsson, R. Julin, S. Juutinen, A. Herzan, J. Konki, M. Leino, J. Pakarinen, J. Partanen, P. Peura et al., Phys. Rev. C 91, 061304(R) (2015).

[41] A. P. Zuker, Phys. Rev. C 103, 024322 (2021).

[42] C. Qi, Phys. Rev. C 94, 034310 (2016).

[43] M. G. Procter, D. M. Cullen, C. Scholey, P. Ruotsalainen, L. Angus, T. Back, B. Cederwall, A. Dewald, C. Fransen, T. Grahn, P. T. Greenlees, M. Hackstein, U. Jakobsson, P. M.
Jones, R. Julin, S. Juutinen, S. Ketelhut, M. Leino, R. Liotta, N. M. Lumley et al., Phys. Rev. C 86, 034308 (2012).

[44] Z. Dombrádi, B. M. Nyako, G. E. Perez, A. Algora, C. Fahlander, D. Seweryniak, J. Nyberg, A. Atac, B. Cederwall, A. Johnson, A. Kerek, J. Kownacki, L. O. Norlin, R. Wyss, E. Adamides, E. Ideguchi, R. Julin, S. Juutinen, W. Karczmarczyk, S. Mitarai et al., Phys. Rev. C 51, 2394 (1995).

[45] R. Machleidt, Phys. Rev. C 63, 024001 (2001).

[46] M. Hjorth-Jensen, T. T. S. Kuo, and E. Osnes, Phys. Rep. 261, 125 (1995).

[47] C. Qi and Z. X. Xu, Phys. Rev. C 86, 044323 (2012).

[48] R. Wyss, J. Nyberg, A. Johnson, R. Bengtsson, and W. Nazarewicz, Phys. Lett. B 215, 211 (1988).

[49] W. Nazarewicz, R. Wyss, and A. Johnson, Nucl. Phys. A 503, 285 (1989). 